\title{
AVALIAÇÃO DAS PROPRIEDADES FÍSICAS E MECÂNICAS DO ADOBE (TIJOLO DE TERRA CRUA)
}

\author{
Evaluation of physical and mechanical properties of adobe bricks
}

\author{
Andréa Aparecida Ribeiro Corrêa ${ }^{1}$,Vitor Hugo Teixeira ${ }^{2}$ \\ Sebastião Pereira Lopes ${ }^{3}$, Marcelo Silva de Oliveira ${ }^{4}$
}

\begin{abstract}
RESUMO
Com a finalidade de verificar a viabilidade do tijolo de terra crua (adobe) na execução de moradias, realizou-se pesquisa para identificar as características físicas e mecânicas do solo e definição de procedimentos e métodos de produção do mesmo, estabelecendo critérios que melhorem a qualidade do produto final. O solo escolhido foi o LATOSSOLO VERMELHO - AMARELO Distrófico, que é característico da Região Sul de Minas Gerais. Teores de umidade e correção granulométrica com areia média $(0,25-0,50 \mathrm{~mm})$ foram adotados segundo Hernandez et al. (1983) e Pacheco \& Dias Júnior (1990). A produção seguiu dois processos, um deles com blocos de terra comprimida (BTC), utilizando prensa manual modelo MRC-1 TECMOR, no tamanho denominado AP $(23 \times 11 \times 5,0 \mathrm{~cm})$ e o outro artesanal, com formas de madeira, denominado BTA, nos tamanhos BA (23 x $11 \times 5,5 \mathrm{~cm})$, CA $(29 \times 14 \times 10 \mathrm{~cm})$ e DA $(29 \times 14 \times 14 \mathrm{~cm})$. Após a fabricação, os adobes foram submetidos a ensaios de resistência à flexão e compressão, com monitoramento de peso, volume, peso específico e umidade em três períodos distintos de "cura" ( secagem) que foram 7, 21 e 35 dias, sendo colocados em cômodo fechado $(\mathrm{F})$, galpão coberto $(\mathrm{G})$ e em local desprotegido sujeito às intempéries (T). Para análise dos dados, optou-se pelo delineamento experimental inteiramente casualizado no esquema fatorial, com quatro repetições, adotando-se o teste de Tukey com nível de significância de 5\%. A produção de BTC não apresentou resultados satisfatórios quando comparada com a produção de BTA. O tamanho BA apresentou os melhores resultados para resistência à flexão e compressão. A estabilização com areia média nos solos argilosos proporcionou a produção de adobes com menor contração. $O$ galpão coberto é ideal para a cura. A resistência à compressão atingiu valores superiores aos encontrados na bibliografia consultada. Concluiu-se que a produção de adobes seguindo os critérios definidos na pesquisa como umidade adequada, estabilização com areia média $(0,25-0,50 \mathrm{~mm})$, utilização de "maromba", cuidados na produção e cura, resulta em tijolos de qualidade superior tanto em resistência como no aspecto estético, comprovando sua viabilidade como alvenaria na construção.
\end{abstract}

Termos para indexação: Adobe, tijolo ecológico, construção com terra.

\begin{abstract}
Aim at verifying the feasibility of the adobe brick for housing construction, a research was carried out to evaluate the mechanical and physical characteristics of the material and to define production procedures and methods, establishing therefore the criteria to improve the product quality. The soil chosen was distrofic (Red - Yellow) Latosoil that is typical in south region of Minas Gerais State. Humidity levels and granulometric correction with medium sand $(0.25-0.50 \mathrm{~mm})$ were adopted according to Hernandez et al. (1983) and Pacheco \& Dias Júnior (1990). The production followed two processes, one of them with compressed dirt blocks (CDB) using manual press MCR-1 TECMOR, called as AP $(23 \times 11 \times 5,0 \mathrm{~cm})$ and another handicraft wood mold brick called BTA size of the bricks are: BA $(23 \times 11 \times 5.5 \mathrm{~cm}), \mathrm{CA}(29 \times 14 \times 10 \mathrm{~cm})$ e DA $(29 \times 14 \times 14 \mathrm{~cm})$. After the manufacturing, the adobes were submitted to flexion and compression resistance tests, with weight, volume, density and humidity monitoring, in three drying distinct periods, which were ranged in seven, twenty-one and thirty-five days. After the adobes were placed in a close room, a shed and an unprotected place subject to weather changes. To analyze the data, a factorial design was used with four replications, with the use of the Tukey test at 5\% of error level. The production of BTC did not present satisfactory results when compared with the production of BTA. The BA size presented the best results for compression and flexion resistance. The stabilization with medium sand in clay soils provides the production of adobes with minor contraction. The shed protection is ideal for the cure process. The resistance to compression achieved higher levels compared to the ones found in the bibliographic references. We concluded that the production of adobe following the criteria defined in the research, such as appropriate humidity, medium sand stabilization, "maromba", the care during production and drying, resulted in bricks of superior quality, concerning resistance and aesthetic aspects proving their feasibility in masonry construction.
\end{abstract}

Index terms: Adobe, ecologic brick, earth construction.

(Recebido para publicação em 28 de abril de 2004 e aprovado em 1 de abril de 2005)

\section{INTRODUÇÃO}

Diante da preocupação atual com construções ecologicamente corretas, ecovilas e bioarquitetura, o adobe surge como importante opção em edificações de baixo custo, por economizar em transporte, quando a terra adequada encontra-se disponível no próprio local da obra; não requerer mão-de-obra especializada; possuir excelente

\footnotetext{
${ }^{1}$ Engenheira Civil, Ms. em Engenharia Agrícola, Construções Rurais e Ambiência, Departamento de Engenharia da Universidade Federal de Lavras/ UFLA - Cx. P. 3037 - 37200-000 - Lavras/MG. deiacor@ufla.br

2 Professor Dr. Adjunto IV do Departamento de Engenharia da Universidade Federal de Lavras/UFLA.

${ }^{3}$ Professor Dr. Adjunto IV do Departamento de Engenharia da Universidade Federal de Lavras/UFLA.

${ }^{4}$ Professor Dr. Adjunto IV do Departamento de Ciências Exatas da Universidade Federal de Lavras/UFLA.
} 
conforto térmico; e não consumir energia para sua fabricação. O consumo de água para a sua produção é sessenta vezes menor que para a do cimento. Não gera vapores contaminantes e não apresenta perigo na sua manipulação. No entanto, trata-se de um processo artesanal que, dependendo de sua composição em contacto com água, sofre desgaste e erosão.

Considerando o grande déficit habitacional e a busca de materiais não poluentes, renováveis e de baixo custo em contrapartida aos industrializados, que têm muitas vezes alto consumo de energia e são centralizadores, é de fundamental importância que essa tecnologia seja resgatada, porque além de gerar trabalho e cidadania, possibilita a integração do profissional com a comunidade em projetos de auto construção e parcerias como, por exemplo, a Engenharia Pública.

A composição granulométrica ideal da mistura de solo para o adobe é de quantidades de argila e silte entre 9 e 3\% em peso, segundo Velloso et al. (1985). Martinez (1979) define $20 \%$ de argila e 40 a $55 \%$ de areia. Alves (1985) considera argila menor que $20 \%$ e areia superior a $45 \%$. Já Hernandez et al. (1983) definem a porcentagem ideal de areia para os adobes próxima de $50 \%$, silte, $30 \%$ e argila, $20 \%$, portanto é fundamental que o solo seja analisado por meio de testes de campo e laboratório para verificar a necessidade ou não da correção granulométrica com areia.

As dimensões empregadas para a produção artesanal são bem variadas. Ortega (1983) refere-se a 29 x 9 x $9 \mathrm{~cm}$ e 30 x 15 x $15 \mathrm{~cm}$ usados em Benin, África. Milanez (1958) cita adobes de $40 \times 20 \times 10 \mathrm{~cm}$ com 12,6 kg e $25 \times 12$ x $8 \mathrm{~cm}$ com $2,5 \mathrm{~kg}$. No México, as medidas usuais são $38 \mathrm{x}$ $38 \times 8 \mathrm{~cm}$ e $39 \times 18 \times 18 \mathrm{~cm}$ e $40 \times 20 \times 15 \mathrm{~cm}$, como cita Williams-Ellis \& Eastwick-Field (1950). Rodrigues (1980) define $30 \times 15 \times 15 \mathrm{~cm}$; o arquiteto egípcio Hassan Fathy indica $25 \times 15 \times 6 \mathrm{~cm}$; Lavinsky et al. (1998) usaram formas dos tamanhos $23 \times 11 \times 7 \mathrm{~cm} \mathrm{e} 23 \times 11 \times 10 \mathrm{~cm}$ em pesquisa desenvolvida no CEPLAC (Centro de Pesquisas do Cacau em Itabuna).

As formas são confeccionadas em madeira em vários modelos e tamanhos (duplas, simples, com encaixe, com $1 / 2, \mathrm{e}^{3 / 4}$ de tijolo), retangulares e quadradas, mas todas com "agarraderas" (apoios laterais para as mãos) nos extremos, para facilitar a produção e o desforme. Algumas são feitas com encaixe "macho-fêmea", que aumenta a aderência quando as paredes são erguidas.

Martinez (1979) e Milanez (1958) consideram de fundamental importância a homogeneização da mistura, que pode ser feita com os pés, ou utilizando a "maromba" ou "pipa". Para a produção de BTC, segundo Picchi et al.
(1986), a quantidade ideal de água na mistura corresponde à umidade ótima $h_{o t^{t}}$. A determinação da $h_{o t}$ é feita por meio de curvas de compactação com energia normal, utilizando a prensa modelo MRC-1 da TECMOR (ABNT, 1986). Para a produção de BTA, Velloso et al. (1985) consideram a quantidade de água entre 7 e $8 \%$ em solos arenosos e 16 e 18\% nos argilosos, enquanto Martinez (1979) especifica entre 12 e $16 \%$ do peso. Devido à complexidade das características físicas e químicas do solo a umidade ideal é muito variável.

Velloso et al. (1985) alertam para a importância da origem mineralógica do material fino componente da mistura de solo. O argilo mineral é responsável pelo grau de expansibilidade do solo. Argilas com alto teor de óxido de ferro e baixo caulinítico tendem a ser pouco expansivas. Observando essas características no material de solo evitase, assim, o fenômeno de contração, que pode contribuir para o aparecimento de trincas e microfissuras durante o processo de cura. Pacheco \& Dias Júnior (1990) determinaram a contração linear para os solos em estudo em seu estado natural e corrigidos com areia, utilizando o método descrito por Enteich \& Augusto (1963), sendo os resultados encontrados utilizados nesse trabalho.

Quanto ao acréscimo de fibras na mistura, há divergências. Neumann et al. (1984), em estudo da resistência à compressão, com palha na mistura em quantidades ideais, afirmam que a mesma controla a microfissuração. Já Milanez (1958) descarta sua utilização, definindo como ineficaz, em razão do apodrecimento do material com o tempo, o que promove a formação de canalículos, diminuindo a resistência dos tijolos. Freire \& Ramirez-Sarmiento (1997) utilizaram fibras de bagaço de cana-de-açucar desmeduladas mecanicamente, in natura e tratadas quimicamente com silicato de sódio combinado com sulfato de alumínio, misturadas à argamassa de cimento e areia. Observaram que a resistência à compressão simples do compósito foi reduzida com o aumento do teor de fibras na mistura e que o tratamento químico aumentou significativamente a resistência mecânica do material obtido.

Inúmeros trabalhos vêm sendo desenvolvidos com a mistura dos mais diversos estabilizantes como cimento, cloreto de cálcio, borra de carbureto, cinzas de bagaço de cana e outros. Rolim \& Freire (1998), que estudaram o aproveitamento do material solo-vinhaça concentrada e sua possível utilização na fabricação de tijolos prensados (BTC), encontraram resultados de resistência à compressão de 1,92 MPa para solo arenoso e 1,7 MPa para solos argiloso. 
Lavinsky et al. (1998), em experimento com BTC desenvolvido em Uruçuca, região cacaueira no sul da Bahia, utilizaram cinza, casca e mel de cacau, e esterco de vaca. O acréscimo de cinza em $30 \%$ nos solos foi a opção que apresentou melhores resultados de resistência à compressão, com 1,67 MPa.

Objetiva-se assim com esta pesquisa determinar as características físicas e mecânicas nas diferentes granulometrias e composições dos solos escolhidos, analisando a estabilização com areia, umidade ideal, tamanhos, procedimentos na produção, resistências à compressão e flexão, tendo como suporte variáveis acessórias, como peso, volume, peso específico e umidade, com a finalidade de confeccionar adobes de melhor qualidade.

\section{MATERIAL E MÉTODOS}

A fase experimental foi conduzida nas dependências do Departamento de Engenharia da Universidade Federal de Lavras - UFLA. O município de Lavras localiza-se na região sul do Estado de Minas Gerais com latitude $21^{\circ} 45^{\prime} \mathrm{S}$, e longitude $45^{\circ} 00^{\prime} \mathrm{W}$, altitude média de 918 metros e precipitação anual de $1493 \mathrm{~mm}$. Foi escolhido o LATOSSOLO VERMELHOAMARELO Distrófico, por ser comum na região sul do Estado e típico do Brasil. Os materiais de solo utilizados foram caracterizados quanto à composição granulométrica, textural, estados de consistência e contração linear (PACHECO \& DIAS JÚNIOR, 1990). Utilizou-se também areia média $(0,25-$ $0,50 \mathrm{~mm}$ ) para atingir as porcentagens de $40 \%$ e $60 \%$ de areia nos materiais de solo.

Seguindo os critérios estabelecidos, que são as quantidades de solo em seu estado natural e corrigidos com areia média $(0,25-0,50 \mathrm{~mm})$, adotou-se as dimensões,

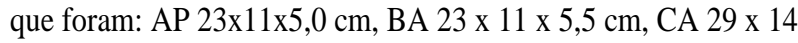
x $10 \mathrm{~cm}$ e DA 29 x 14 x $14 \mathrm{~cm}$, conforme mostra a Figura 1.

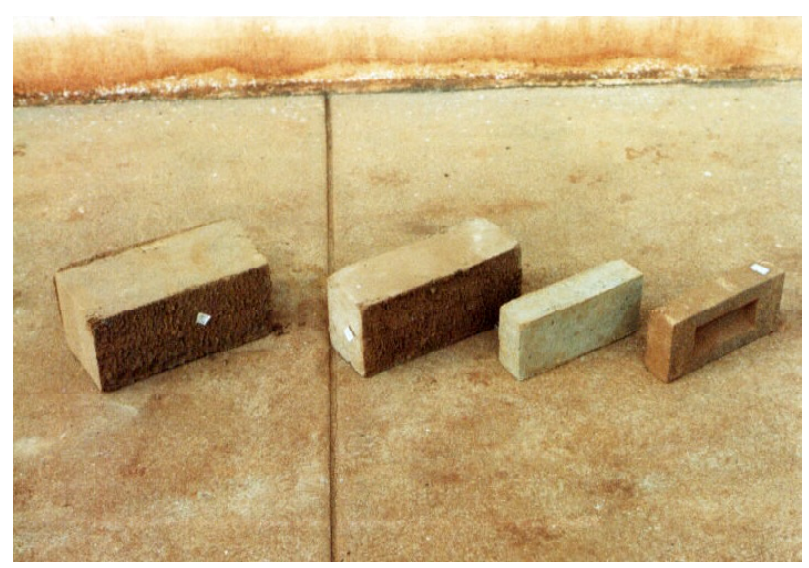

FIGURA 1 - Tamanhos BTA (DA,CA,BA) e BTC (AP), respectivamente.
Todos os solos foram submetidos ao peneiramento com peneira \# 4 (4,76 mm). As correções granulométricas foram feitas em peso com o solo completamente seco, seguindo procedimento definido por Pacheco \& Dias Júnior (1990), para 60\% no solo LVAdm (LATOSSOLO VERMELHOAMARELO Distrófico textura média) e LVAdarg (LATOSSOLO VERMELHO- AMARELO Distrófico textura argilosa), e 40\% e 60\% para o LVAdmarg (LATOSSOLO VERMELHO-AMARELO Distrófico textura muito argilosa).

Dois processos de produção foram definidos: a fabricação de BTC (bloco de terra comprimida), utilizada para solo-cimento, solo-cal e outras misturas estabilizadas, denominada produção mecânica, e de BTA (bloco de terra artesanal) que é a produção artesanal tradicional, com a mistura dos componentes do adobe feita na "maromba" para melhor homogeneização. Para BTC adotou-se a umidade ótima determinada por Pacheco \& Dias Júnior (1990) por meio do ensaio de compactação descrito por Picchi et al. (1986), com a quantidade de água determinada em peso, e o umedecimento do material de solo com regador, com 6,5 $\mathrm{kg}$ de mistura para cada três unidades. Para BTA, adotou-se a umidade determinada pelo método de campo descrito por Hernandez et al. (1983), cujos resultados foram a média de três repetições, com a quantidade de água medida em litros, seguindo os teores determinados para cada mistura, e umedecimento do material de solo com regador. A produção foi dividida para três locais definidos: cômodo fechado $(\mathrm{F})$; galpão coberto (G); e no tempo (T), sujeito a intempéries. Os adobes foram submetidos aos ensaios de resistência à flexão e compressão. Para este trabalho foram analisados os resultados obtidos aos 35 dias de cura. Antes dos ensaios de compressão e flexão, determinou-se pesos e medidas de cada amostra, observando-se o aparecimento de fissuras e o aspecto estético da produção como cantos bem definidos e qualidade final do bloco. Os ensaios de compressão e flexão seguiram os procedimentos segundo Enteich \& Augusto (1963). Após os ensaios foi coletada amostra para determinação de umidade. Optou-se pela utilização da prensa manômetro para o ensaio de flexão, quando os adobes não rompiam com a máxima carga possível pelo método citado acima. Os resultados do Ensaio de Contração Linear (PACHECO \& DIAS JÚNIOR, 1990) foram comparados com nova leitura nas amostras das caixas de madeira, feita após seis meses, com a finalidade de verificar se ocorreram aumento no número de fissuras e na contração.

A composição granulométrica dos solos utilizados em seu estado natural, determinada por Pacheco \& Dias Júnior (1990) foi comparada com a composição ideal para os adobes (HERNANDEZ et al., 1983), como observa-se na Figura 2. 


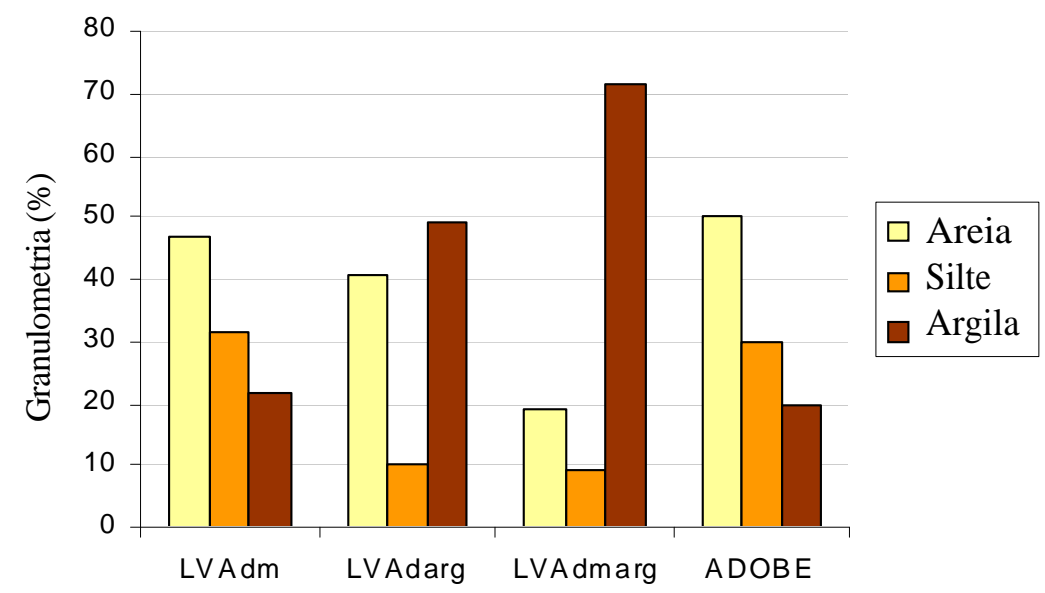

FIGURA 2 - Granulometria dos solos em estudo comparada à ideal para o adobe.

O delineamento experimental determinou nove combinações de tipos de solos e locais. Cada uma dessas combinações gerou um experimento isolado ou independente planejado e conduzido no esquema fatorial. Os fatores definidos para cada experimento foram: correção, tamanho e cura. O fator tamanho teve como níveis AP, BA, CA e DA. O fator correção granulométrica teve como níveis: natural com $0 \%$ de correção e $60 \%$ para LVAdm e LVAdarg; e para o LVAdmarg os níveis natural, $40 \%$ e $60 \%$. O fator cura teve como níveis 7, 21 e 35 dias. As variáveis principais estudadas foram compressão e flexão. Todos os tratamentos foram feitos com quatro repetições. Foi adotado para a comparação o teste de Tukey com o software SANEST, cujos autores são Elio Paulo Zonta e Amauri Almeida Machado do Instituto Agronômico de Campinas - IAC. O nível de significância considerado foi de 5\%. Na Tabela 1 exemplifica-se os fatores considerados pelo delineamento experimental para o LVAdm, que seguiu o mesmo critério para o LVAdarg e para o LVAdmarg com o acréscimo do nível $40 \%$.

\section{RESULTADOS E DISCUSSÃO}

Conforme a Figura 2, observa-se que o LVAdm é o solo cuja composição granulométrica mais assemelha-se ao adobe. Esse solo pode ser utilizado em seu estado natural, simplificando-se os procedimentos na produção. Comparando-se o LVAdmarg com o LVAdarg observa-se que ambos necesssitam de correção com areia em quantidades distintas. Conhecer a granulometria do solo é um procedimento essencial para a produção de adobes porque, além de quantificar os componentes do material solo, define a necessidade ou não da estabilização com areia.
Pela Tabela 2, observa-se que a umidade ideal para o BTC, determinada pela curva de compactação na prensa MRC-1 Tecmor, quando comparada aos resultados obtidos pelo ensaio proposto por Hernandez et al. (1983) para produção de BTA, apresentou teores inferiores, em média duas vezes menores. Cada mistura de solo requer uma quantidade específica de água, portanto não é possível definir um teor de umidade padrão para a confecção de adobes. Pode-se inferir que nos solos em estudo quando acrescentou-se areia média, a quantidade de água necessária diminuiu em $30 \%$ para o BTC e $20 \%$ para o BTA.

Quanto à contração linear, foi feita nova leitura após seis meses, constatando que os solos argilosos apresentaram maiores variações dimensionais com o decorrer do tempo, e que o acréscimo de areia contribuiu para a estabilização dos solos LVAdarg e LVAdmarg, que mantiveram o mesmo número de trincas e variação menor de contração, como pode ser observado na Tabela 3. As trincas estabilizaram-se, exceto para o LVAdmarg em seu estado natural, devido ao alto teor de argila em sua composição. Todos os materiais de solo apresentaram índice de contração menor que o admitido, que é de 5,08 cm (ENTEICH \& AUGUSTO, 1963).

À medida que os adobes foram estabilizados com areia, o peso aumentou e o número de vazios diminuiu, conforme Tabela 4, determinando um produto final mais estável e de melhor qualidade para os solos arenosos. O tamanho CA apresentou maior aumento em peso no solo LVAdmarg quando comparado com os outros solos, ficando DA, que tem maior espessura, sem variação significativa. Pode-se inferir que a espessura do adobe influenciou na eficiência da correção granulométrica com areia, principalmente nos solos mais argilosos, com melhores resultados nos adobes de menor espessura.

Ciênc. agrotec., Lavras, v. 30, n. 3, p. 503-515, maio./jun., 2006 
TABELA 1 - Delineamento experimental para o LVAdm em função da correção com areia (\%), cura (dias) e tamanho (cm).

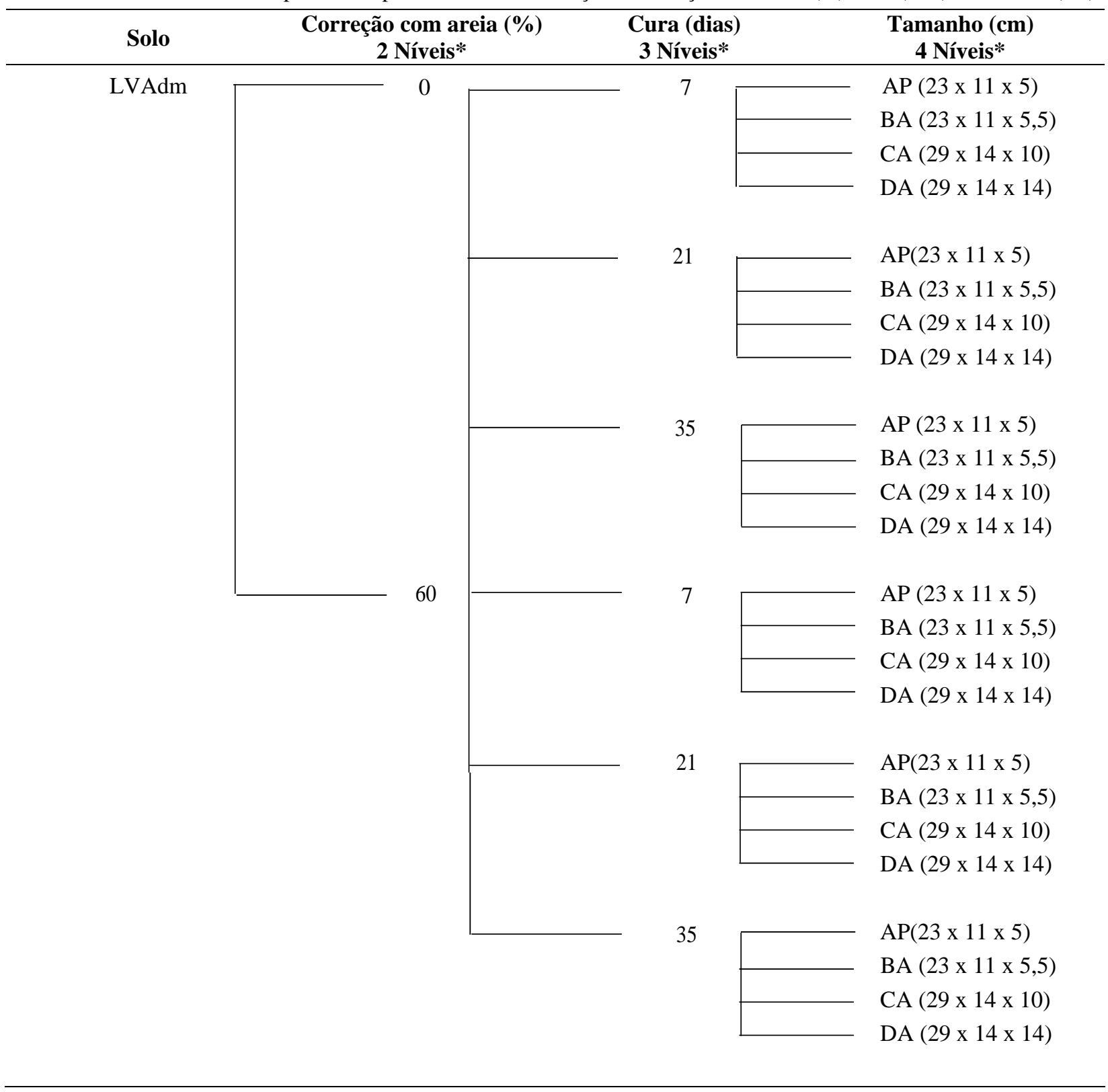

$* 2 \times 3 \times 4 \times 4$ repetições $=24$ Tratamentos. 
TABELA 2 - Umidade ideal para produção de adobes BTC e BTA de acordo com o tipo de solo e o nível de correção com areia $(\%)$.

\begin{tabular}{cccc}
\hline \multirow{2}{*}{ Solos } & $\begin{array}{c}\text { Correção com areia } \\
(\boldsymbol{\%})\end{array}$ & $\begin{array}{c}\text { Umidade }(\boldsymbol{\%}) \\
\text { BTC }\end{array}$ & $\begin{array}{c}\text { Umidade (\%) } \\
\text { BTA }\end{array}$ \\
\hline \multirow{2}{*}{ LVAdm } & 0 & 12,00 & 27,10 \\
& 60 & 8,80 & 22,35 \\
LVAdarg & 0 & 16,00 & 34,76 \\
& 60 & 11,60 & 27,17 \\
LVAdmarg & 0 & 28,40 & 48,53 \\
& 40 & 19,60 & 38,19 \\
& 60 & 13,20 & 30,04 \\
\hline
\end{tabular}

TABELA 3 - Contração linear dos solos.

\begin{tabular}{lccccc}
\hline \multirow{2}{*}{ Solos } & \multirow{2}{*}{ Correção $(\boldsymbol{\%})$} & \multicolumn{2}{c}{$\mathbf{7}$ dias } & \multicolumn{2}{c}{ 6 meses } \\
\cline { 3 - 6 } & & Contração $(\mathbf{c m})$ & Trincas & Contração $(\mathbf{c m})$ & Trincas \\
\hline \multirow{2}{*}{ LVAdm } & 0 & 1,58 & 3 & 2,18 & 3 \\
\multirow{2}{*}{ LVAdarg } & 60 & 0,40 & 7 & 0,52 & 7 \\
& 0 & 1,80 & 2 & 2,80 & 2 \\
LVAdmarg & 60 & 0,96 & 2 & 1,30 & 2 \\
& 0 & 0,78 & 2 & 1,80 & 4 \\
& 40 & 0,40 & 1 & 0,40 & 1 \\
& 60 & 0,16 & 0 & 0,30 & 0 \\
\hline
\end{tabular}

TABELA 4 - Peso dos adobes aos 35 dias no galpão coberto.

\begin{tabular}{lccccc}
\hline \multirow{2}{*}{ Solos } & \multirow{2}{*}{ Correção $(\%)$} & \multicolumn{4}{c}{ Peso $(\mathbf{k g f})$} \\
\cline { 3 - 6 } & & AP & BA & CA & DA \\
\hline LVAdm & 0 & 2,051 & 2,191 & 6,143 & 8,760 \\
\multirow{2}{*}{ LVAdarg } & 60 & 1,930 & 2,415 & 6,549 & 9,180 \\
& 0 & 1,678 & 1,814 & 5,416 & 8,995 \\
LVAdmarg & 60 & 1,833 & 2,041 & 6,235 & 9,144 \\
& 0 & 1,486 & 1,563 & 4,945 & 6,669 \\
& 40 & 1,522 & 1,665 & 5,461 & 7,460 \\
& 60 & 1,808 & 1,930 & 5,934 & 8,720 \\
\hline
\end{tabular}

A variação volumétrica foi maior nos solos argilosos, como pode ser observado na Tabela 5, demonstrando novamente a propriedade da areia em preencher os vazios, diminuindo a porosidade. Para o BTC ocorreu menor variação volumétrica quando comparado ao BTA, exceto para o LVAdmarg, devido a sua composição granulométrica que apresenta grande porcentagem de argila. Os solos arenosos apresentaram os maiores volumes.

Ciênc. agrotec., Lavras, v. 30, n. 3, p. 503-515, maio./jun., 2006 
Quanto ao peso específico observou-se que para o tamanho DA do LVAdarg os resultados foram inferiores mesmo com a correção granulométrica, sugerindo que a composição do solo e a espessura maior inibiram a ação estabilizadora da areia.

A umidade apresentou maiores variações nos solos mais argilosos, conforme Tabela 7, porque esses retêm mais água. O resultado de umidade aos 35 dias, no local galpão coberto, determinou que nesse período de cura os adobes encontram-se em condições de uso para construção.

Para a resistência à compressão, a interação entre os fatores tamanhos, correção e cura foi significativa entre o LVAdm, LVAdarg e LVAdmarg conforme a Tabela 8. Tanto para os solos no estado natural quanto para os estabilizados, o tamanho BA apresentou resultados superiores, destacandose dos demais, sendo que o tamanho AP, produzido com a prensa manual, apresentou os piores resultados, exceto para o solo LVAdm no estado natural. A correção granulométrica foi mais eficiente para CA quando comparada a DA, por ter mesmas dimensões e menor espessura.
A interação entre os fatores tamanho, correção e cura foi significativa conforme pode ser observado na Tabela 9. Para os tamanhos BA e CA , a correção granulométrica apresentou melhores resultados. A estabilização com areia, apesar dos resultados em alguns casos ser inferior ao estado natural dos solos, proporcionou principalmente no LVAdarg e LVAdmarg melhor qualidade dos adobes, diminuindo a perda na produção devido à ocorrência de trincas e fissuras. Para o tamanho AP, a correção granulométrica apresentou resistências menores com relação ao estado natural para todos os solos. Para BA, observa-se um melhor resultado da correção com areia para o LVAdmarg, que mantem a resistência e diminui a probabilidade de contração. Para CA, observou-se o melhor resultado em todas as misturas de solos, com acréscimo na resistência para LVAdm e LVAdarg, e para LVAdmarg, a correção com $60 \%$ apresentou resultados superiores a de $40 \%$. Com o tamanho DA, quando comparado com CA, demonstrou-se que a espessura maior teve influência na perda de resistência do adobe, quando estabilizado.

TABELA 5 - Volume dos adobes aos 35 dias no galpão coberto.

\begin{tabular}{cccccc}
\hline \multirow{2}{*}{ Solos } & \multirow{2}{*}{ Correção $(\%)$} & \multicolumn{4}{c}{ Volume $\left(\mathbf{c m}^{\mathbf{3}}\right)$} \\
\cline { 3 - 6 } & & AP & BA & CA & DA \\
\hline LVAdm & 0 & 1.170 & 1.259 & 3.515 & 4.956 \\
& 60 & 1.175 & 1.337 & 3.848 & 5.002 \\
LVAdarg & 0 & 1.153 & 1.115 & 3.228 & 4.563 \\
& 60 & 1.181 & 1.183 & 3.461 & 4.901 \\
LVAdmarg & 0 & 1.072 & 1.097 & 3.172 & 4.207 \\
& 40 & 1.153 & 1.172 & 3.335 & 4.580 \\
& 60 & 1.175 & 1.183 & 3.486 & 4.826 \\
\hline
\end{tabular}

TABELA 6 - Peso específico dos adobes aos 35 dias no galpão coberto.

\begin{tabular}{lccccc}
\hline \multirow{2}{*}{ Solos } & \multirow{2}{*}{ Correção $(\%)$} & \multicolumn{4}{c}{ Peso específico $\left(\mathbf{k g f} / \mathbf{c m}^{\mathbf{3}}\right)$} \\
\cline { 3 - 6 } & & AP & BA & CA & DA \\
\hline LVAdm & 0 & 1,753 & 1,740 & 1,704 & 1,769 \\
& 60 & 1,643 & 1,803 & 1,702 & 1,835 \\
LVAdarg & 0 & 1,455 & 1,626 & 1,678 & 1,971 \\
& 60 & 1,552 & 1,726 & 1,827 & 1,869 \\
LVAdmarg & 0 & 1,269 & 1,424 & 1,559 & 1,585 \\
& 40 & 1,321 & 1,421 & 1,637 & 1,629 \\
& 60 & 1,539 & 1,631 & 1,702 & 1,807 \\
\hline
\end{tabular}


TABELA 7 - Umidade dos adobes aos 35 dias no galpão coberto.

\begin{tabular}{cccccc}
\hline \multirow{2}{*}{ Solos } & \multirow{2}{*}{ Correção $(\boldsymbol{\%})$} & \multicolumn{4}{c}{ Umidade $\mathbf{( \% )}$} \\
\cline { 3 - 6 } & 0 & AP & BA & CA & DA \\
\hline LVAdm & 60 & 0,97 & 0,93 & 1,10 & 0,97 \\
& 0 & 1,84 & 1,35 & 0,42 & 0,81 \\
LVAdarg & 60 & 1,37 & 2,22 & 2,29 & 2,02 \\
& 0 & 2,40 & 1,31 & 1,93 & 1,53 \\
LVAdmarg & 40 & 2,76 & 2,43 & 5,15 & 3,94 \\
& 60 & 1,91 & 1,89 & 2,76 & 1,88 \\
& & & & 1,68 & 2,02 \\
\hline
\end{tabular}

TABELA 8 - Resistência à compressão aos 35 dias de cura no galpão coberto fixando correção.

\begin{tabular}{cccccc}
\hline \multirow{2}{*}{ Solos } & \multirow{2}{*}{ Correção $(\%)$} & \multicolumn{4}{c}{ Resistência à compressão (MPa) } \\
\cline { 2 - 5 } & & AP & BA & CA & DA \\
\hline LVAdm & 0 & $2,06^{\mathrm{a}}$ & $2,38^{\mathrm{a}}$ & $1,88^{\mathrm{a}}$ & $1,63^{\mathrm{a}}$ \\
& 60 & $0,71^{\mathrm{c}}$ & $2,63^{\mathrm{a}}$ & $1,75^{\mathrm{b}}$ & $1,63^{\mathrm{b}}$ \\
LVAdarg & 0 & $1,25^{\mathrm{c}}$ & $4,00^{\mathrm{a}}$ & $1,88^{\mathrm{c}}$ & $2,75^{\mathrm{b}}$ \\
& 60 & $1,00^{\mathrm{c}}$ & $2,38^{\mathrm{b}}$ & $3,44^{\mathrm{a}}$ & $1,88^{\mathrm{b}}$ \\
LVAdmarg & 0 & $1,75^{\mathrm{c}}$ & $3,38^{\mathrm{b}}$ & $4,50^{\mathrm{a}}$ & $3,38^{\mathrm{b}}$ \\
& 40 & $1,38^{\mathrm{c}}$ & $3,50^{\mathrm{a}}$ & $2,50^{\mathrm{b}}$ & $2,50^{\mathrm{b}}$ \\
& 60 & $1,06^{\mathrm{c}}$ & $3,21^{\mathrm{a}}$ & $2,81^{\mathrm{a}}$ & $1,88^{\mathrm{b}}$ \\
\hline
\end{tabular}

Médias com letras iguais na horizontal não diferem entre si, com nível de significância de 5\%, pelo Teste de Tukey

TABELA 9 - Resistência à compressão aos 35 dias de cura no galpão coberto fixando tamanho.

\begin{tabular}{ccccc}
\hline \multirow{2}{*}{ Solos } & Tamanhos & \multicolumn{3}{c}{ Resistência à compressão (MPa) } \\
\cline { 3 - 5 } & AP $(23 \times 11 \times 5 \mathrm{~cm})$ & $2,06^{\mathrm{a}}$ & $\mathbf{6 0} \%$ & $\mathbf{4 0} \%$ \\
\hline LVAdm* & BA $(23 \times 11 \times 5,5 \mathrm{~cm})$ & $2,38^{\mathrm{a}}$ & $2,71^{\mathrm{b}}$ & \\
& CA $(29 \times 14 \times 10 \mathrm{~cm})$ & $1,88^{\mathrm{a}}$ & $1,75^{\mathrm{a}}$ & \\
& DA $(29 \times 14 \times 14 \mathrm{~cm})$ & $1,63^{\mathrm{a}}$ & $1,63^{\mathrm{a}}$ & \\
LVAdarg* & AP & $1,25^{\mathrm{a}}$ & $1,00^{\mathrm{a}}$ & \\
& BA & $4,00^{\mathrm{a}}$ & $2,38^{\mathrm{b}}$ & $1,38^{\mathrm{a}}$ \\
& CA & $1,88^{\mathrm{b}}$ & $3,44^{\mathrm{a}}$ & $3,00^{\mathrm{b}}$ \\
LVAdmarg & DA & $2,75^{\mathrm{a}}$ & $1,88^{\mathrm{b}}$ & $2,50^{\mathrm{a}}$ \\
& AP & $1,75^{\mathrm{a}}$ & $1,06^{\mathrm{a}}$ & $2,50^{\mathrm{b}}$ \\
& BA & $3,38^{\mathrm{a}}$ & $3,25^{\mathrm{b}}$ & \\
& CA & $4,50^{\mathrm{a}}$ & $2,81^{\mathrm{b}}$ & $1,88^{\mathrm{b}}$ \\
& DA & $3,38^{\mathrm{a}}$ &
\end{tabular}

Médias com letras iguais na horizontal não diferem entre si, com nível de significância de 5\%, pelo Teste de Tukey.

* Para estes solos não houve o nível 40\% de correção.

Ciênc. agrotec., Lavras, v. 30, n. 3, p. 503-515, maio./jun., 2006 
Nas Figuras 3, 4 e 5 estão representados, respectivamente, os resultados de resistência à compressão para o LVAdm, LVAdarg e LVAdmarg. Analisando os resultados obtidos no galpão coberto, observa-se que, aos 35 dias, o LVAdm (N) apresentou pouca variação para todos os tamanhos, ao passo que o LVAdm (60) teve o tamanho BA destacando-se dos demais, com o resultado de 2,63 MPa. A estabilização não apresentou vantagens para esse solo, exceto para BA. Os tamanhos maiores, CA e DA, mais estáveis, não apresentaram variações significativas quando comparados com o solo em seu estado natural e corrigido com areia. Para o LVAdarg $(\mathrm{N})$, novamente BA apresentou melhor resultado, com 4,00 MPa, e para LVAdarg
(60), o tamanho CA, com 3,44 MPa. Os resultados obtidos no estado natural apresentaram maiores variações. A estabilização para o tamanho CA foi mais eficiente que para DA, devido à sua espessura ser menor. O LVAdarg apresentou-se mais instável nos tamanhos maiores. Para o LVAdmarg (N), o tamanho CA com 4,50 MPa foi o melhor resultado; para o LVAdmarg (40), BA destacou-se com 3,50 $\mathrm{MPa}$, e para LVAdmarg (60), a variação entre BA, com 3,21 $\mathrm{MPa}$, e CA com 2,81MPa, não foi significativa. O LVAdmarg apresentou maior variação nos resultados em seu estado natural, sendo o tamanho CA mais beneficiado pela estabilização, por ter espessura menor que DA. Os tamanhos maiores mostraram-se mais instáveis.

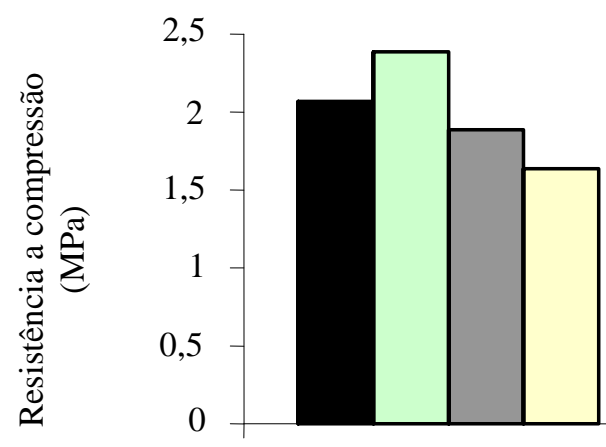

natural

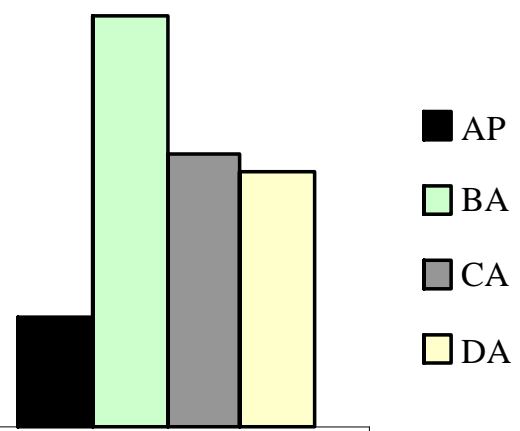

60

Correção granulométrica(\%)

FIGURA 3 - Resistência à compressão do LVAdm aos 35 dias de cura no galpão coberto.

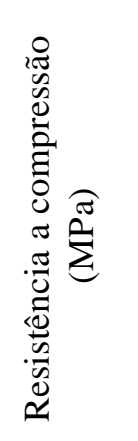

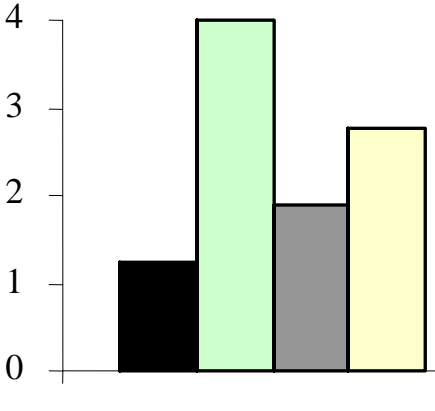

natural

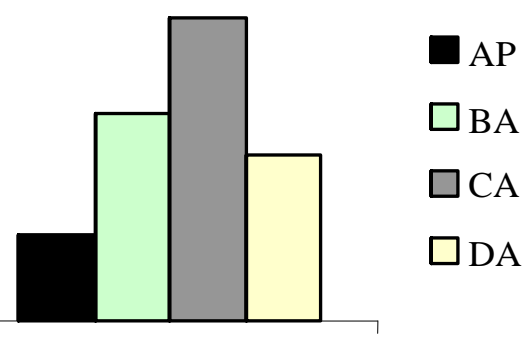

60

Correção granulométrica (\%)

FIGURA 4 - Resistência à compressão do LVAdarg aos 35 dias em galpão coberto. 


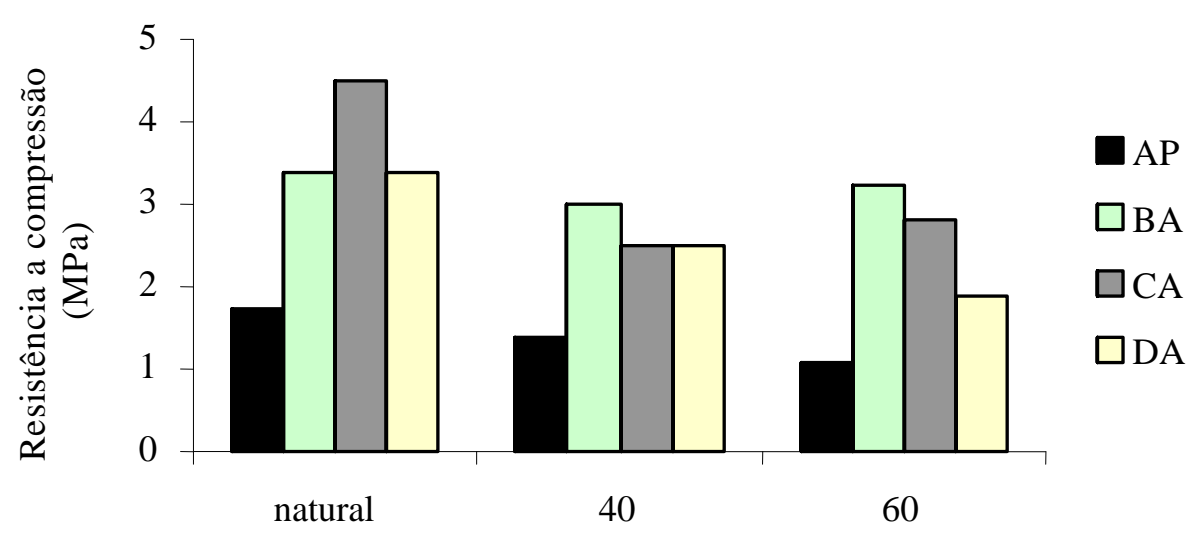

Correção granulométrica (\%)

FIGURA 5 - Resistência à compressão do LVAdmarg aos 35 dias em galpão coberto.

Para a resistência à flexão, a interação entre os fatores tamanho, correção e cura foi significativa, exceto para o LVAdarg no cômodo fechado e no galpão coberto conforme pode ser observado na Tabela 10 . O ensaio de resistência à flexão demonstrou que para o LVAdm , o LVAdarg e o LVAdmarg, tanto no estado natural como corrigido com areia média, os melhores resultados apresentados foram para o tamanho BA e para LVAdmarg.

Conforme a Tabela 11, cujos resultados obtidos pelos desdobramentos em interações duplas e triplas estão relacionados, a interação entre os fatores tamanho, correção e cura foi significativa, exceto para o LVAdarg no cômodo fechado e no galpão coberto. Observa-se que à medida que os tamanhos aumentam, a correção com areia mostra-se mais eficiente, equilibrando os resultados de resistência. Observou-se que DA apresentou resultado superior de resistência à flexão em relação à CA devido a sua maior espessura, sendo a estabilização mais eficiente para esses tamanhos. Para os tamanhos CA e DA, a correção granulométrica determinou melhora na resistência à flexão, principalmente nos adobes que ficaram no tempo, contribuindo para menor contração do solo. O melhor resultado foi para o tamanho BA. A estabilização para DA foi mais eficiente que para CA. Para o LVAdarg(N) e LVAdarg (60) a correção granulométrica apresentou os mesmos resultados para todos os locais de cura. Pelos resultados observa-se para CA e DA, a eficiência da correção com areia. Para o LVAdmarg (N) e LVAdmarg (40) e LVAdmarg (60) os tamanhos AP e BA, quando comparados o estado natural com a correção granulométrica, observa-se que novamente a resistência à flexão foi inferior para os solos estabilizados. O contrário é demonstrado para os tamanhos CA e DA.
Nas Figuras 6, 7 e 8 estão representados, respectivamente, os resultados de resistência à flexão para o LVAdm, LVAdarg e LVAdmarg no galpão coberto. Pelos resultados observa-se para CA e DA, a eficiência da correção com areia. Para o LVAdmarg (N) e LVAdmarg (60) os tamanhos AP e BA, quando comparados o estado natural com a correção granulométrica, observa-se que novamente a resistência à flexão foi inferior para os solos estabilizados. O contrário é demonstrado para os tamanhos CA e DA.

\section{RESULTADOS E DISCUSSÃO}

Em razão da grande quantidade de informações abordadas nesta pesquisa, são feitas as considerações seguintes, com a finalidade de elucidar os caminhos que levarão às conclusões:

- O processo de produção de adobes é artesanal, sendo o material de solo seu principal componente. Portanto, fazem-se necessários a análise granulométrica e estudo do nível de expansão da argila a princípio, e dos demais testes referidos no estudo de Pacheco \& Dias Júnior (1990).

- O processo de produção BTC sem estabilização com produtos como DS-328, cinza, cimento e outros, apresentou resultados duas vezes inferiores demonstrando que esse método não é adequado para a produção do adobe, que utilizou apenas areia média como correção granulométrica e água em quantidade ideal.

- A quantidade de água acrescentada na mistura é em função do tipo de solo a ser utilizado. Quanto mais argiloso, maior necessidade de água. 
- O processo artesanal que utiliza a "maromba" para a mistura do material de solo, conferiu melhor resistência aos adobes, por proporcionar maior homogeneização da mistura.

- Os resultados de resistência à compressão encontrados foram superiores à média referida na bibliografia consultada, que variou de $0,5 \mathrm{MPa}$ para BTA e $18 \mathrm{MPa}$ para BTC.

- Os resultados médios de resistência à flexão correspondem a aproximadamente $30 \%$ dos resultados de compressão.

- A produção em galpão coberto possibilita a perda gradual de água, contribuindo para melhor qualidade do adobe.
- O tamanho BA (23 x $11 \times 5,5 \mathrm{~cm})$ superou as expectativas, demonstrando que adobes robustos não são sinônimo de maior resistência.

- Comparando-se resultados de CA (29 x 14 x $10 \mathrm{~cm})$ e DA ( 29 x 14 x $14 \mathrm{~cm})$, observa-se que o primeiro tamanho tem resistências superiores ao segundo, sugerindo que menor espessura proporciona blocos de melhor resistência à compressão. $\mathrm{O}$ oposto ocorreu para a resistência à flexão.

- O peso, volume, peso específico e umidade final contribuiram como variáveis acessórias para a análise e discussão dos resultados.

- A estabilização com areia média foi mais eficiente para os solos argilosos.

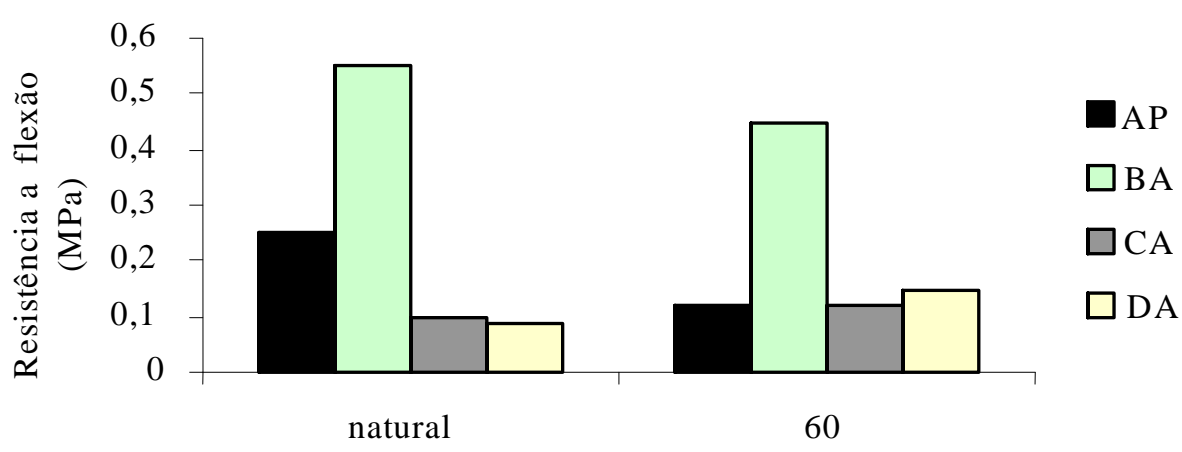

Correção granulométrica (\%)

FIGURA 6 - Resistência à flexão do LVAdm aos 35 dias em galpão coberto.

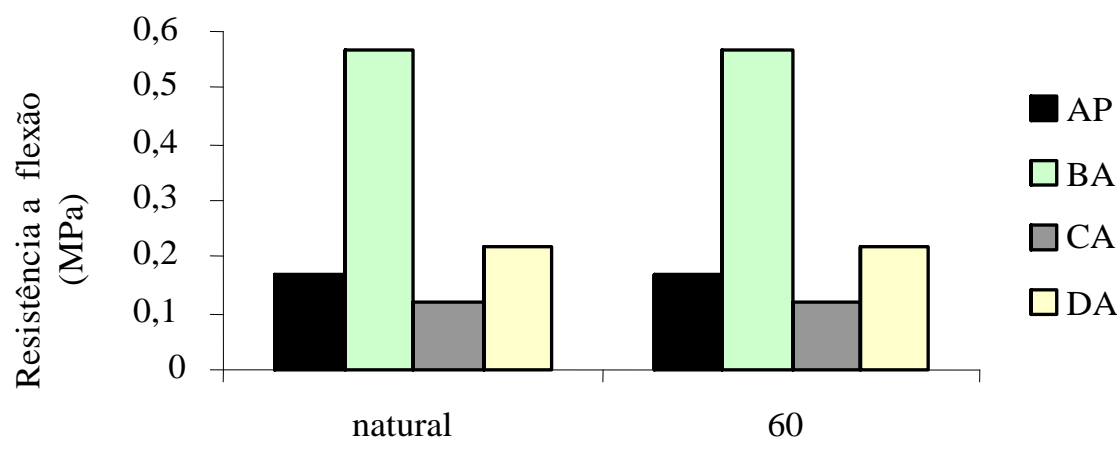

Correção granulométrica (\%)

FIGURA 7 - Resistência à flexão do LVAdarg aos 35 dias em galpão coberto. 


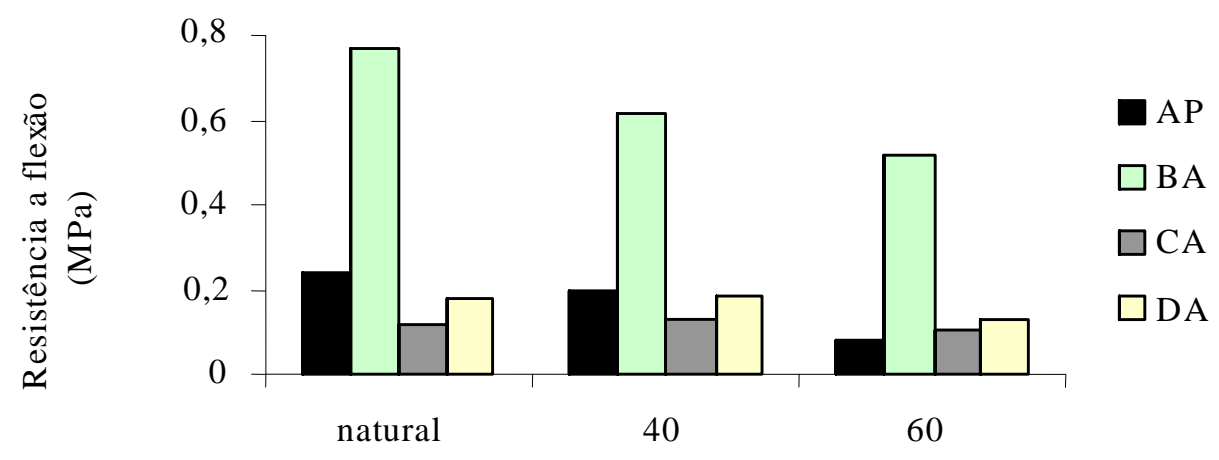

Correção granulométrica (\%)

FIGURA 8 - Resistência à flexão do LVAdmarg aos 35 dias em galpão coberto.

\section{CONCLUSÕES}

O processo de produção de BTA (bloco de terra artesanal) apresentou resultados superiores quando comparado à produção de BTC (bloco de terra comprimida), para produção de adobes em estado natural e estabilizados com areia média, optando-se, então, pelo primeiro processo. O local de cura ideal para o adobe é o galpão coberto, por proporcionar perda gradual de umidade e maior uniformidade na secagem, evitando-se assim o fenômeno da contração e o aparecimento de trincas, que diminuem a resistência e o aproveitamento da produção.

A espessura dos adobes nos tamanhos CA $(29 \times 14$ x $10 \mathrm{~cm})$ e DA $(29$ x 14 x $14 \mathrm{~cm})$ influenciou os resultados de resistência à flexão e compressão. Quanto maior espessura, menor resistência à compressão, e maior a resistência à flexão. A estabilização com areia média proporcionou adobes de melhor qualidade, principalmente nos tamanhos maiores CA e DA. O tamanho BA $(23 \times 11 \times 5,5 \mathrm{~cm})$ apresentou os melhores resultados de resistência à flexão e compressão.

Os critérios adotados na produção referentes à umidade ideal e estabilização com areia média promoveram a melhor qualidade no produto final. Todo material de solo deve ser caracterizado quanto à granulometria e composição mineralógica para que seja determinada a sua viabilidade para produção de adobes.

\section{REFERÊNCIAS BIBLIOGRÁFICAS}

ALVES, A. T. Terra tierra earth terre. [S.1.: s.n.], [1985?]. Não paginado. Trabalho acadêmico encontrado no Arquivo do Departamento de Materiais de Construção da Escola de Arquitetura da UFMG.
ASSOCIAÇÃO BRASILEIRA DE NORMAS TÉCNICAS. NBR 7182: solo-ensaio de compactação: método de ensaio. Rio de Janeiro, 1986. 10 p.

ENTEICH, G.; AUGUSTO, A. Suelo-cimento su application en la edificacion. Bogotá: Centro Interamericano de Vivienda y Planeamiento, 1963.99 p.

FREIRE, W. J.; RAMIREZ-SARMIENTO, C. Argamassa de cimento e areia combinada com fibras de bagaço de cana-de-açucar. Engenharia Agrícola, Jaboticabal, v. 17, n. 2, p. 1-8, dez. 1997.

HERNANDEZ, R.; ENRIQUE, L.; LUNA, M. L. A. Cartilha de pruebas de campo para seleccion de tierras en la fabricación de adobes. México: Conescal, 1983. 72 p.

LAVINSKY, E. C. A.; SERÔDIO, R. S.; FERREIRA FILHO, E. de M.; CUNHA, J. Resistência de adobes estabilizados com diversos materiais disponíveis na região cacaueira da Bahia. In: CONGRESSO BRASILEIRO DE ENGENHARIA AGRÍCOLA, 19., 1998, Piracicaba, SP. Anais... Piracicaba: CEPLAC/CEPEC/EMARC, 1998.

MARTINEZ, E. A. (Coord.). Manual para la construcion das viviendas con adobe. [S.l.: s.n.], 1979. 16 p. (Cuadernos de Material Didático, 2).

MILANEZ, A. Casa de terra, as técnicas de estabilização do solo a serviço do homem do campo. São Paulo: SESP, 1958.

NEUMANN, J. V.; BERNALES, J. B.; BLONDET, M. Resistência sísmica de la mamposteria de adobe. Lima: Pontifícia Universidade Católica del Peru, 1984. 19 p. (Publicacion 01-84.01. Série Difision). 
ORTEGA, A. Materiaux et techniques de construction. Lima: Pontifícia Universidad Católica del Peru, 1983. $43 \mathrm{p}$.

PACHECO, A. A. R. C.; DIAS JÚNIOR, M. S. Estudo comparativo de métodos de campo e laboratório aplicados à confecção de blocos em adobe. [S.l.: s.n.], 1990. $14 \mathrm{p}$.

PICCHI, F. A.; CINCOTTO, M. A.; BARROS, T. M. C. Tijolos de solo cal. A Construção, São Paulo, n. 2017, p. 25-30, out. 1986.

RODRIGUES, J. W. A casa de moradia no Brasil antigo. Revista do Serviço de Patrimônio Histórico e Artístico Nacional, São Paulo, p. 159-197, 1980.
ROLIM, M. M.; FREIRE, W. J. Resistência à compressão de tijolos fabricados com solo-vinhaça concentrada. Engenharia Agrícola, Jaboticabal, v. 17, n. 3, p. 1-8, mar. 1998.

VELLOSO, C. H. V.; LARA, D. S.; FARIA NETO, J. L.; SAFFAR, J. M. E.; PEREIRA, N. T. Z. Relatório parcial de acompanhamento do projeto "estabilização de solos por processos físicos e físicos-químicos para a construção de paredes de alvenaria ou monolíticos em habitações unifamiliares de baixo custo". Belo Horizonte: CETEC, 1985. $14 \mathrm{p}$.

WILLIAMS-ELLIS, C.; EASTWICK-FIELD, E. Building in cobpise, and stabilized earth. London: Country Life, $1950.138 \mathrm{p}$. 